\title{
TUMOR DE BRENNER
}

Descripción de un caso de localización extra-ovariana :

\author{
Dr. Hernando Latorre L.
}

\begin{abstract}
Nota de la Dirección
Por considerarlos de gran interés científico, y por cortesía especial de los autores y de los "Anales de la Sociedad de Biología de Bogotá”, reprocucimos estos dos artículos sobre Tumor de Brenner con los debidos agradecimientos.
\end{abstract}

El Tumor de Brenner, llamado así por Sobert Meyer en 1932, quien además lo reconoció como un tipo nuevo de neoplasma especial del ovario, es un tumor fibro-epitelial con zonas de aspecto mucoide y de allí el nombre de fibro-epitelioma mucoide (Plaut). Es de ocurrencia rara y con frecuencia es hallazgo quirúrgico o de autopsia, por su tendencia a ser de tamaño pequeño o por estar asociado a otra alteración, clínicamente más notoria. Antes de su reconocimiento como entidad neoplásica distinta y de su separación por Meyer, de los tumores de células granulosas, algunos tumores ováricos que corresponden a la estructura brenneriana, fueron interpretados como carcinomas primitivos o metastásicos o asimilados a otras variedades neoplásicas. Según Kerpe (11) y col. el primer caso, fue reportado por Mac Naughton-Jones en 1898, quien lo llamó Adenofibroma. Orthann, un año después, describió otro caso bajo el nombre de Fibroma papilar superficial carcinomatoso. Con el nombre de carcinoma sólido, Fothergill y Clockner publicaron tres casos. Fritz Brenner en 1907 describió tres casos del tumor llamado por él Ooforoma folicular, pues anota que los islotes epiteliales semejan folículos ováricos y el estroma lo equipara al conjuntivo tecal. Novak (17) anota que

\footnotetext{
* Presentado a la Sociedad de Biología en la sesión de abril 10 de 1955.
} 
Frankl reportó, con el nombre de Fibroadenoma adenoquístico, un grupo de tumores que se habían considerado como fibromas con agregaciones epiteliales, diferentemente interpretadas.

Resumen de la historia clínica.-Caso del doctor Alberto Villaneda S. Hospital de San José.

J. V.-Mujer de sesenta y cinco años de edad. Menarquia a los diez y ocho años. Un embarazo a término con parto normal, hace 30 años. Hace 12 años le fue practicada una miomectomía y después fue tratada con radium, para metropatía hemorrágica; desde entonces está en amenorrea. Hace tres años aparece hernia umbilical. Desde seis meses antes de su ingreso al hospital presenta metrorragias mensualmente con duración de tres días, semejantes a sus antiguas menstruaciones. Ha notado crecimiento progresivo del abdomen.

Al examen clínico, como datos al respecto, se encuentra: abdomen globuloso, hernia umbilical de contenido epiploico. Tumor redondeado abdominal cuyos límites no se alcanzan a definir por résistencia que ofrece la enferma. El hipogastrio está ocupado por una masa blanda, poco móvil, dolorosa. Vulva entreabierta. Periné relajado pero continente, vagina estrecha. Utero: cuello aparece atrofiado y por su orificio escapa sangre oscura. La cúpula vaginal está ocupada por una masa dura, dolorosa al tacto. Ovarios. No se palpan. Recto y ano: ligero rectocele y cistocele.

De los varios exámenes de laboratorio, anotamos uno de líquido ascítico que dio el siguiente resultado: Rivalta: negativo. B. de Koch: negativo. Se encontraron células tumorales malignas.

Se practicó una intervención quirúrgica en la cual se extirpó un fibromioma uterino, que en los cortes histológicos mostró marcada hialinización. En el acto quirúrgico se encontró en el epiplón mayor infiltración de aspecto tumoral maligno, confirmándose histológicamente la presencia de un adenocarcinoma metastásico de origen endometrial. También se extirpó una trompa uterina que conserva su forma y mide 6 x 0.7 centímetros y adherida a ella por tejido flojo hay un nódulo, que el cirujano pudo observar completamente independiente del ovario respectivo; es de superficie lisa, de consistencia firme, con un diámetro mayor de 2 centímetros y cuya superficie de sección es de color gris amarillento y muestra varias cavidades pequeñas. Este nódulo presenta la constitución histológica de Tumor de Brenner.

Quince días después fue practicada una histerectomía total con extirpación del resto de los anexos. La matriz mide $9 \times 10 \times 7$ 

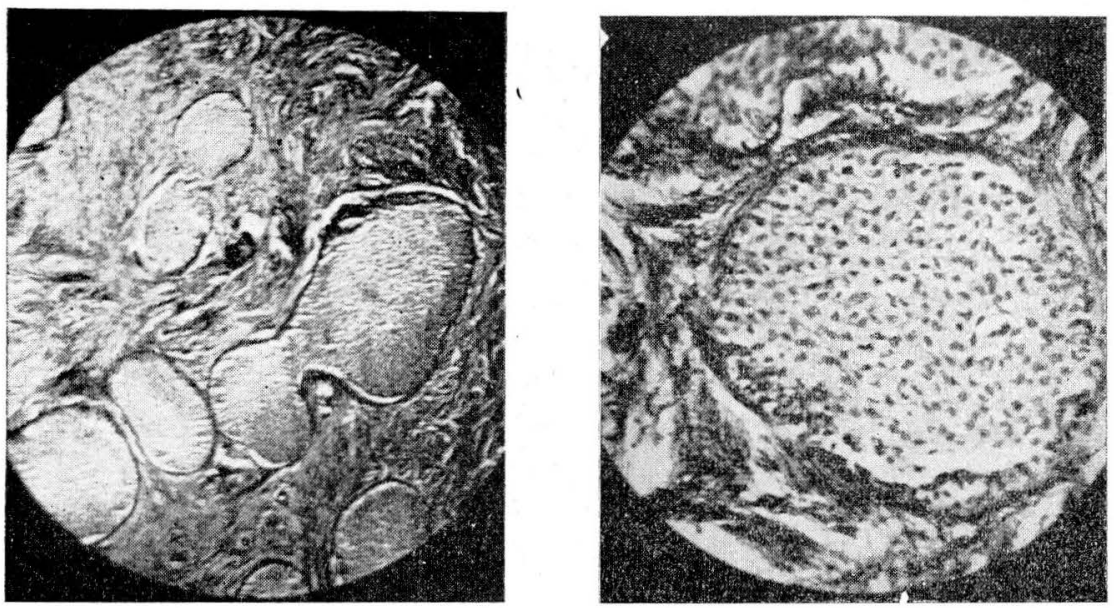

MICRO NUMERO 1.-Arquitectura general del tumor de Brenner. Is?otes epiteliales de varios tamaños, entre tejido fibroso. Col. H. E.

MICRO NUMERO 2.-Masa sólida de células poliédricas, con citoplasma claro y vacuolado; núclecs alargados y triangulares que muestran surco. Col. H. E.

cent metros. El cuerpo está deformado por varios nódulos duros, de superficie de sección fasciculada, el más grande de los cuales tiene un diámetro mayor de 5 centímetros. En la cavidad uterina se encuentra, especiaimente hacia el lado derecho, un tejido frable, de aspecto neoplásico que infiltra el miometrio y se extiende al canal endo-cervical. Viene la trompa derecha, ligeramente flexuosa, mide $6 \times 0.7$ centímetros y el ovario, que mide $3,6 \times 2 \times 1,5$ centímetros. Separadamente viene el ovario izquierdo que mide $3,5 \times 2,2$, x 1,7 centímetros. Queda un resto de trompa izquierda de 1,5 centímetros de longitud. En los cortes histológicos se hace el diagnóstico de fibromiomas, adenocarcinoma endometrial con metástasis a ambos ovarios y en ia superficie de la trompa derecha. El ovario izquierdo, esclerosado lo mismo que el derecho, muestra hemorragia superficial, pero no encontramos imagen brenneriana ni signos que indicasen un posible sitio en relación con el Tumor de Brenner, antes examinado.

\section{COMENTARIOS}

Frecuencia.-El Tumor de Brenner, nombre actualmente aceptado, es de ocurrencia rara. Ackerman (1) en 1953 anota como descritos 175; sin embargo, Kerpe (11) en 1952 recuerda el tra- 
pajo de Graccone sobre 319 casos encontrados en la literatura hasta 1946. Entre nosotros solamente he encontrado referencia al hallazgo de un caso por López Escobar (12), (13). Es posible que por la falta de exámenes histopatológicos rutinarios, tumores de este tipo hayan pasado inadvertidos y considerados como otra variedad, especialmente como fibromas simples.

Edad.-La mayor incidencia se encuentra en la post-menopausia. Willis (18) anota la estadística de Fox sobre 158 casos en la cual encontró 120 entre la $5^{\text {a }}$ y $8^{a}$ décadas; 26 en ia 4 a y 12 en la $2^{\text {a }}$ y $3^{\text {a }}$

Situación.--El Tumor de Brenner se ha descrito prácticamente siempre inciuido y en relación con el tejido ovárico, sea hacia la zona cortical o hacia la medular y el hilio, o haciendo saliente de la superficie ovárica, mostrando superficialmente alguna cantidad de estroma de la glándula. Algunos autores y precisamente como argumento en contra de la interpretación histogenética que io considera derivado de los islotes de Walthard, anotan que a pesar de ser estos agregados epiteliales mucho más trecuentes en situaciones fuera del ovario, como en la superficie de las trompas uterinas o ligamentos, no se han encontrado tumores en estas localizaciones. El presente caso se encontró, en la intervención quirúrgica, sin relación inmediata con el ovario e histológicamente no se encontró evidencia de ningún tejido propio a este órgano en los cortes correspondientes al tumor. El Tumor de Brenner puede también aparecer en la pared de cistadenomas serosos y más generalmente pseudomucosos, en forma de un nódulo más o menos grande o el tumor en general puede tener la estructura quística, correspondiendo a las dos formas definidas por Ewing (6): sólidos y quísticos. En la mayoría de los casos publicados el Tumor de Brenner es unilaterai como lo anota Curtis y col (4). Willis (18) considera que puede ser bilateral en un $8 \%$ de los casos.

Benignidad.-En general se considera como tumor benigno, especialmente en su forma sólida, ya que como lo anota Novak, a lo menos dos casos que se han publicado con evolución maligna, han correspondido a formas quísticas (Dubrauszky, citado también por Kerpe) y otro caso, perteneciente a distinto tipo histológico que fue hallado por Limburg (17). Además Ewing (6) y Navarro (16) citan un caso con recidiva (Tavildoroff) y otro de muerte por metástasis (Blaud, Goldstein). 
Significación hormonal.-Está hoy aceptado que el Tumor de Brenner no es funcionante. En los casos con hemorragias postmenopáusicas se han encontrado otras lesiones capaces de producirias como pólipos, fibromas, carcinomas, etc., o tumoraciones pélvicas como cistadenomas ováricos o tamaño grande del tumor que por hiperhemia generalizada de la pelvis o por sustancias elaboradas en las células ovarianas de la pared del quiste, la pueden explicar (Kerpe). En muchos casos no se ha examinado el endometrio o se ha encontrado atrófico. Solamente se encuentra referencia de hiperplasia endometrial atribuída a la presencia del Tumor de Brenner en publicaciones de Schiffman en 1932 y de Pendl en 1948, Novak-Kerpe (17) (11). Otros casos de metrorragias post-menopáusicas en enfermas con Tumor de Brenner y con hiperplasia endometrial pueden atribuírse a la asociación de pequeños tumores de células granulosas, que pueden haber pasado inadvertidos o a fuente extra-ovariana de hormonas con acción estrogénica.

Aspecto macroscópico.-Los Tumores de Brenner tienden a ser pequeños, pero pueden adquirir un tamaño bastante grande, pero siempre de crecimiento lento, caso en el cual pueden presentar sintomatología en relación con su tamaño y a veces acompañarse con ascitis incluso con hidrotórax, constituyendo el "Sindrome de Meigs". Frecuentemente se observa en ovarios que tienen otra clase de tumor como teratoma o tumor de células granulosas y se encuentra también en asocio con otro tumor del aparato genital benignos o malignos, como fibromiomas o carcinomas uterinos o tubáricos y también con endometriosis. Como se anotó anteriormente, Ewing considera dos tipos fundamentales: sólidos y quisticos. Foot (8) describe otra variedad, la microquistica y de pequeñas cavidades quísticas llenas por material mucoide claro, de aspecto semejante a "queso suizo". Una forma semejante se encontró en el caso que se describe.

Los tumores sólidos y de quistes pequeños adquieren un menor tamaño que los quísticos; son de consistencia firme, esféricos o nodulares, sin tendencia a la necrosis o a hemorragia. No tienen cápsula definida pero con frecuencia muestran alguna cantidad de estroma ovárico en la superficie. Son de un color rosado o gris amarillento, con estrías blanquecinas y son más o menos aparentes las pequeñas cavidades de pared interior lisa y de contenido mucoide. 
Los tumores quísticos pueden corresponder a la morfología de un quiste pseudomucoso o seroso en cuya pared aparece incluído el tejido brenneriano. Cierto número de casos puede corresponder al desarrollo del cistadenoma pseudomucoso en un Tumor de Brenner ya que en ellos se puede observar desde la transformación pseudomucosa del epitelio en los islotes o en pequeñas cavidades, hasta la estructura completa del cistadenoma. También el predominio desmoplásico puede llegar a la constitución de fibromas sólidos en los cuales hay apenas presencia ocasional de acúmulos epiteliales. El est'mulo de esa proliferación conjuntiva que puede llegar a constituir la mayor parte del tumor, es desconocido, pero como dice Faulker (7) hay reacciones similares en otros tumores del ovario como por ejemplo en los tumores de Krukenberg y aun de tejido más especializado, como el tejido semejante al linfoide que rodea algunos islotes de endometriosis.

Estructura microscópica.-La arquitectura general del neoplasma consiste en nidos y cordones de células epiteliales, de forma globulosa o cilíndrica, de diferentes tamaños, situados dentro de un estroma fibroso denso, que puede presentar hialinización y a veces calificación. Tal estructura general se muestra en la micro número 1.

Se anota por Geist (9) y Muliigan (15) la ausencia de conectivo y de retículo en el interior de las masas celulares. Barzilai (3) describe siete tipos de células epiteliales que pueden presentarse en la constitución de tales nidos o cordones y que él ciasifica, en las tres primeras variedades, como células de epitelio paramalpighiano o de epitelio brenneriano. Estos tres primeros ripos corresponden a "indiferenciado" cuando son células con núcleo central alargado basofílico y escaso citoplasma; de tipo simplemente "paramalpighiano o de Brenner" cuando son de límites bien distintos, redondas o poliédricas con núcleo central basófilo y el citoplasma abundante ligeramente acidófilo y el tipo -regresivo o adulto" cuando las células son de límites netos grandes, poliédricas, con núcleo vesicular, con contenido variable de cromatina y de forma alargada o a veces triangular y el citoplasina abundante claro y ocasionalmente vacuolado. Además de estos tres tipos que son los distintivos, hay otros no constantes y a veces raros, que corresponden a células poliédricas grandes con queratinización, a células redondeadas con capacidad para pro- 


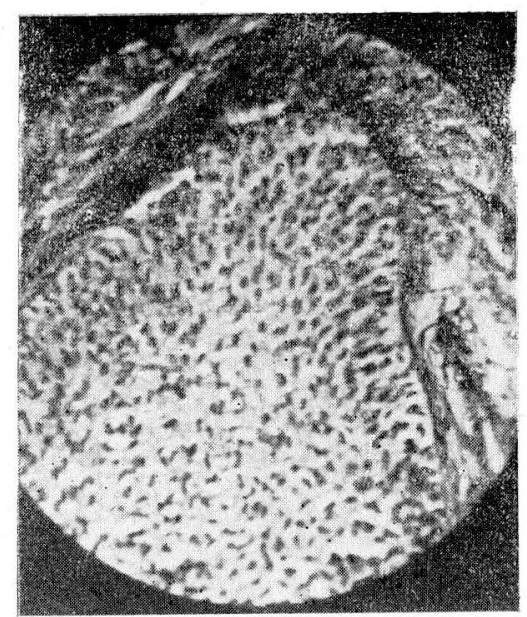

MICRO NUMERO 3.-Masa celular de tipo transicional, con células periféricas en disposición radiada. Se observa el estroma que rodea. Col. H. E.

aucir sustancia mucoide, a células columnares con citoplasma apical vacuolado y núcleo basal semejante a las constitutivas de los cistadenomas pseudo-mucosos y por último a células aplanadas que para Barzilai semejan ciertos aspectos de epitelio seioso. Los tres primeros tipos celulares son los que se encuentran con mayor frecuencia y en el caso presente se pueden distinguir las dos últimas de estas variedades, es decir, agrupamientos constituídos por células poliédricas con núcleo central basófilo y citoplasma abundante ligeramente coloreado o claro y núcleos alargados o triangulares en los cuales se observa la invaginación o surco que anota Varangot, lo mismo que Danfort (1942) y Arey (1943). Micro número 2.

Según la agrupación y estructura de las céluias epiteliales uue forman las masas tumorales, éstas pueden aparecer como "paramalpighianas indiferenciadas" o como áreas más grandes y columnas ramificadas, en las cuales, según la incidencia del corte, las células periféricas son alargadas dispuestas en sentido radial y hacia el centro redondeadas o poliédricas, adoptando el tipo de masas de epitelio "transicional paramalpighiano". Micro número 3.

Se observan también agregados celulares sólidos en los cuales los elementos son grandes, de límites netos y que presentan un aspecto semejante a pavimentosos. 
La parte central de las masas epiteliaies tienden con frecuencia a mostrar cambios degenerativos de licuefacción, por lo cual se forman cavidades centrales irregulares, sin que haya diIerenciación celular que las tapice, constituyendo pseudo-quistes. Micro número 4.

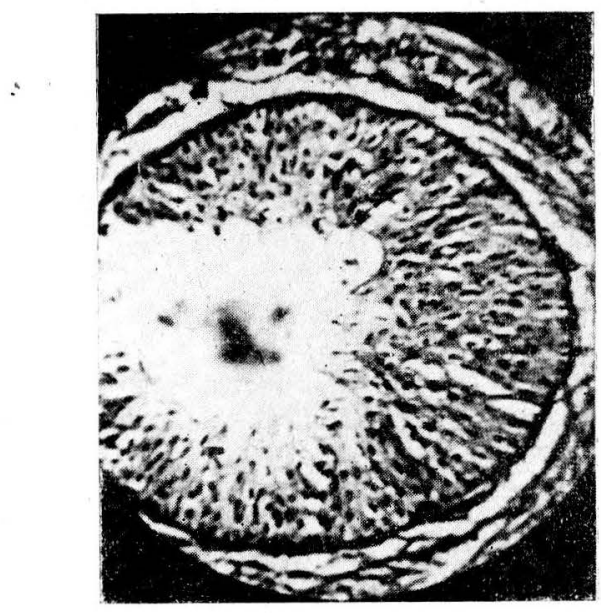

MICRO NUMERO 4.-Masa paramalpighiana con formación de pseudo quiste. Col. H. E.

Estas caviclades contienen un material a veces de aspecto citoplásmico o masas redondas hialinas, a lo cual se debió que fueran interpretadas como semejantes a folículos ováricos o a cuerpos de Call-Exner (Mulligan) (15), y de allí la interpretación y nombre que fue dado por Brenner. Como anota Novak (17), Meyer y otros, observaron que las células epiteliales más internas que tapizan las cavidades centrales, cuando hay la formación de quistes verdaderos, y las cuales pueden ser aplanadas, cúbicas o columnares, muestran sus núcleos periféricos, es decir, lejos de la luz, en tanto que las células que constituyen las estructuras foliculares en la variedad foliculomatosa del tumor de granulosa muestran su núcleo hacia la parte central, por consiguiente más hacia la luz de la cavidad y además en este caso la cubierta epitelial es continua. En las microfotografias números 5 y 6 se muestran cavidades quísticas verdaderas y en una de ellas se aprec1a claramente el epitelio alto que la tapiza, sostenido por una pequeña faja de células brennerianas. 
En las céiulas del Tumor de Brenner se ha demostrado la presencia de glicógeno y de sustancia mucoide y nunca contienen sustancia lípida, la cual sí se encuentra en los foliculomas.

Como se anotó anteriormente, el otro componente histológico del Tumor de Brenner corresponde al tejido fibroso denso con cóiulas fusiformes de tipo adulto, que se distribuye en fascícuios orientados en varios sentidos y localizados alrededor de los nidos epiteliales.

En las variedades quísticas sea que se considere el Tumor de Brenner como localizado en la pared de un cistadenoma o sea que este se forme a expensas de aquél, se encontrarán las cavidades tapizadas por el epitelio columnar característico, con formaciones tubulares y glandulares y en sitios de dichas paredes agregados de tejido brenneriano ya descrito. En la forma más sólida. fibromatosa, se verán masas epitaliales pequeñas, diseminadas en medio de abundante conectivo.

\section{Histogénesis}

La histogéneis del Tumor de Brenner ha sido objeto de muchas interpretaciones, las cuales, sin embargo, pueden reducirse a dos: el origen mesodérmico en varias de sus estructuras de diferenciación y el origen teratomatoso.

Brenner sostuvo primitivamente que los tumores se engendraban en estructuras foliculares por la semejanza que antes se anotó, de sus islotes epiteliales con dichas formaciones y aún Willis (18), al estudiar las difarentes potenciaildades de evolución de que pueden ser capaces los tejidos gonadales y yuxta-gonada-. ies, con producción de estructuras semejantes a las encontradas en algunos tumores de Brenner, especialmente cuando muestran combinaciones cistadenomatosas, cree en la posibilidad de readmitir ese punto de vista original. Geist (9) dice que Stenberg piensa en un desarrollo a expensas de células granulosas. Sin embargo, entre los tumores de Brenner y los de la granulosa no existe semejanza ni funcional ni estructural.

Ia teoría de Meyer es la que se acepta más comúnmente como la que tiene más posibilidades de exactitud. Corresponde al origen de los tumores en los islotes epiteliales descritos primeramente por Ricardo Werth en 1887, y que fueron estudiados por Walthard en 1903 y luego por su discípuio Akagi en 192:, y que son conocidos con el nombre de islotes de Waithard. Se encuen- 

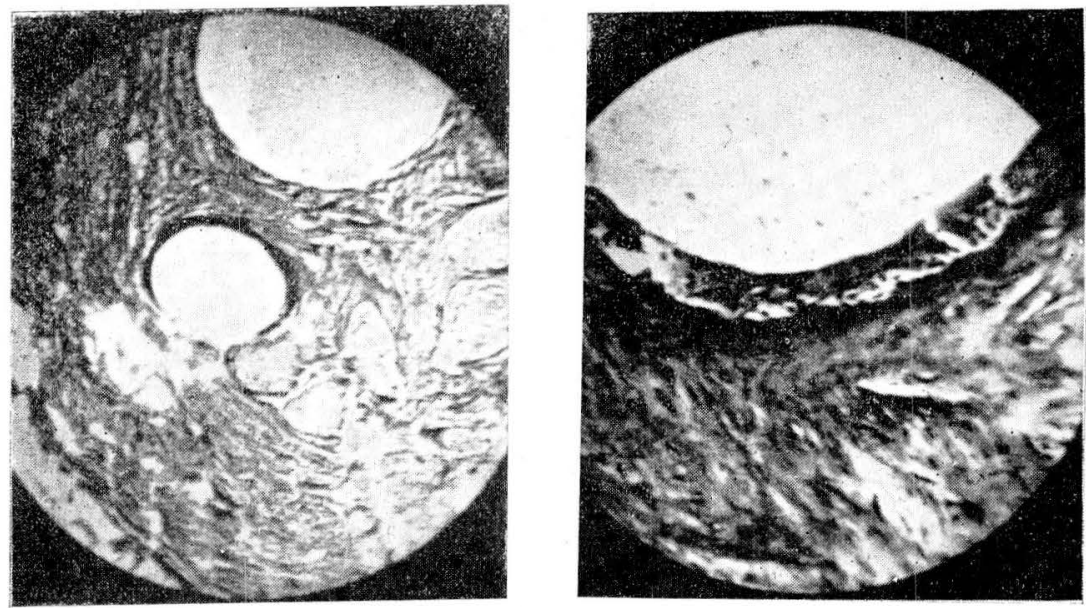

MICRO NUMERO 5.-Se aprecian masas sólidas y tres cavidades quísticas. Col. H. E.

MICRO NUMERO 6.-Cavidad quística revestida por epitelio alto, con núclecs basales y hacia afuera, células Brennerianas. Col. H. E.

tran hacia la superficie del ovario, hacia el hilio y mesosalpinx, con frecuencia en la superficie de la trompa uterina y hacia los iigamentos, sitios en los cuales muchos anotan, son más frecuentes y sin que allí se desarrollen tumores de Brenner, dando esto como argumento en contra de la teoría de Meyer; sin embargo en la cita de Muller que hace Ackerman (1) también pueden dar lugar a tumores brennerianos sólidos.

Los islotes de Walthard se forman por inclusiones metapiásicas del epitelio superficial del ovario, epitelio celómico, el cual en otras condiciones puede mostrar también transformaciones evolutivas semejantes a las que constituyen los tumores de Brenner, como es el caso de las periooforitis crónicas.

Los conductos de Muller se forman por invaginaciones del epitelio celómico, que comienzan en embriones de 10 milímetros (Dodds) (5). Cuando el mesonefro presenta su desarrollo mayor, es decir, tanto los islotes de Walthard como los canales de Muller tienen un origen celómico común. El tejido mulleriano tiene diversas potencialidades de desarrollo y así al tiempo que diferencia mucosas como la tubárica y endometrial, también evoiuciona hacia el tipo mucoso secretor del endocervix, al pavimentoso estratificado de la porción vaginal del cuello y por lo menos a 
parte de la vagina. Kerpe (11) en apoyo del origen mulleriano del Tumor de Brenner anota la posibilidad de que muchos de los tumores endometriales diagnosticados como adenoacantomas, sean en realidad brennerianos. En la historia clínica que acompaña a su trabajo, refiere que por biopsia endouterina se hizo el ciagnóstico histológico de adenoacantoma, por lo cual se practicó histerectomía; se halló luego un Tumor de Brenner ovariano y la imagen histológica del tumor endometrial era perfectamente comparable a la ovárica, sin signos de infiltración miometrial ni de malignidad citológica, por lo cual fueron ambos interpretados como el mismo tipo brenneriano.

Hay una gran semejanza en la estructura celular de los islotes de Walthard con los brennerianos, tanto en la morfología de las células, en su contenido de glicógeno como en la tendencia a la formación quística y pseudo-quística, a la transformación mucoide, etc., evoluciones semejantes también a las presentadas por el epitelio superficial del ovario, que, como hemos recordado, tiene el mismo origen embriológico.

Schiller en su trabajo publicado en 1934 y en el estudio microscópico de uno de sus casos de localización hacia el hilio ovárico encontró transición histológica entre el tejido tumoral y tejidos del "rete ovarii" y entonces interpretó como una persistencia anormal de la relación embrionaria entre el "rete ovarii" y el epófooro. Este se halla formado por tubos paralelos o divergentes localizados en el hilio hacia la trompa, constituídos por epitelio columnar bajo o cúbico, a veces ciliado, rodeado por conectivo y muscular (Maximow) (14). Corresponde a los tubos mesonéfricos que forman unión uro-genital imperfecta, de la cual no se ha de constituir ningún órgano femenino, en tanto que en el hombre forman los conos eferentes y el epididimo. Los tubos mesonéfricos que no entran en esa unión y persisten, constituyen el parooforon, correspondiente al paradidimo en el hombre. Las estructuras halladas hacia el hilio ovárico corresponden propiamente al "rete ovarii" restos de los cordones celulares primitivos del tejido germinal, representados especialmente por las células de Berger, análogas a las de Leydig y llamadas simpático-trópicas y por los restos tubulares del cuerpo de Wolff o mesonefro. Es por lo tanto de este origen mesonéfrico que Schiller cree se derivan algunos tumores de Brenner y halla semejanza morfológica en tales tumores con las imágenes que se pueden encontrar en el epitelio del aparato urinario, como transformación pseudo-mucinosa, estructuras en la carúncula uretral o en 
la pared de la vejiga, en ciertos tipos de cistitis (Faulkner) (7). Esta teoría de Schiller no explicaría el desarrollo tumoral hacia a corteza y además en el hilio ovárico también se encuentran islotes de Walthard.

Al tiempo que se explican las teorías anotadas, los mismos autores aceptan que ei Tumor de Brenner puede tener varios origenes y así, como dice Novak (17), Schiller opina que su teoría no está en pugna con la de Meyer, casi universalmente aceptada, que admite diversidad de origen, según los casos (Greene) (10).

La teoría sobre el origen teratomatoso, es decir, de blastómeras multipotentes que pueden dar iugar a formaciones de diferentes tejidos mesodérmicos, endodérmicos y ectodérmicos, en las cuales sólo aparecerían los componentes brennerianos, habiendo desaparecido los otros (Anderson) (2). La idea de esve origen se basa en la ocurrencia frecuente de la asociación con dermoides, cistadenomas, estruma ovárica y asi Barzilai (3) opina que debe ser considerada esta histogénesis y Meyer también piensa que algunos tumores de Brenner son teratomatosos y se originan en la vida embrionaria (Kerpe) (11).

\section{RESUMEN :}

Se describe y comenta un caso de Tumor de Brenner hallaao por el cirujano, independiente del ovario, en una enferma con adenocarcinoma endometrial. No se observó en dicho tumor ningún tejido ovárico.

\section{BIBLIOGRAFIA}

1. ACKERMANN L. V.-"Surgical Pathology". The C. V. Mosby Co. 1953.

2. ANDERSON W. A. D.- "Pathology". The C. V. Mosby Co. St. Louis. 1948.

3. BARZILAI GEMMA.- "Atlas of Ovarian Tumors". Grune \& Stratton. N. Y. 1943.

4. CURTIS J. F., RACE G. J.-"Bilateral Brenner and Krukenberg Tumors with ovarian Cystadenomas". A. M. A. Arch of Path. 58. 588-591. 1954.

5. DODDS G. S.- "The Essentials of Human Embriology". Jchn Wiley \& Sons N. Y. 1946.

6. EWING J-_"Oncología". Salvat S. A. Barcelona. 1948.

7. FAULKNER R .L., DOUGLAS Ml-"Essentials of Obstetrical and Gynecological Pathology". The C. V. Mosby Co. St. Louis. 1949.

8. FOOT N. CH.-"Pathology in Surgery". J. B. Lippincott Co. Ph. 1945.

9. GEIST S. H.- "Ovarian Tumcrs". Paul B. Hoeber. Inc. N. Y. 1942.

10. GREENER R. R.- "Diverse Origin of Brenner Tumors". Am. J. Obst. \& Gynec. 64,: 878-898. 1952. 
11. KERPE S., BLACK K. M., SPEER F.-"Observations on the Histogenesis of the Brenner Tumor". A. M. A. Arch of Path, 54: 139-148. 1952.

12. LOPEZ ESCOBAR G.-“Tumores del Ovario. Medicina y Cirugía". 1952.

13. LOPEZ ESCOBAR G.--"Virilismos". "Unidia", número 4. Julio. 1953.

14. MAXIMOW A., BLOOM W.-A Textbook of Histology Saunders Co Ph. 1947.

15. MULLIGAN R. M.-"Syllabus of Human Neoplams". Lea \& Febiger. Ph. 1951.

16. NAVARRO. R. M.-"Presentación y comentarios de dos casos infrecuentes en Ginecología: tumor de Brenner del ovario y Sarcoma del endometrio". Arch. Cubanos de Cancerología. 13: 243-251. 1954.

17. NOVAK E.- "Ginecología y Obstetricia". Ed. Alhambra. S. A. Madrid. 1954. 1948.

18. WILLIS R. A.- "Pathology cf Tumors". The C. V. Nosby Co. St. Louis.

\section{DISCUSION}

Doctor G. LOPEZ ESCOBAR

El trabajo del doctor Latorre, profusamente ilustrado, es de positivo interés tanto por la completa descripción histo-patológica que él hace, como por los comentarios sobre frecuencia, histogénesis, etc., que la acompañan. Es de mencionar al referirme a la histogénesis aun no completamente dilucidada del tumor en cuestión, que si bien la mayoría de los autores acogen la opinión de Meyer de que son derivados de ios llamados Islotes de Walthard, la significación verdadera de tales Islotes y su origen mismo no está completamente aclarado tampoco.

En el resumen de la historia existe un examen de laboratoro sobre líquido ascítico, pero en la descripción clínica no existe mención acerca de la existencia de éste ni hay datos sobre si se investigaron metástasis pleurales con el consiguiente hidrotórax, etc. Tratándose de un tumor de tan escasa frecuencia, cuya concomitancia con adenocarcinoma endometrial lo hace aún más raro, tal vez hubiera sido interesante para los clínicos una descripción un tanto más completa sobre el cuadro presentado por esta enferma. En todo caso, si la frecuencia del Brenner Ovárico es escasa, como que sólo hay publicados un poco más de doscientos en la literatura internacional, la incidencia del Brenner extra-ovárica es aún menor, apenas sí una o dos decenas publicados. Si a esto se agrega la concomitancia con el Adenocarcinoma Endometrial, el caso se convierte en un caso extraordinario cuya publicación será una verdadera contribución a nuestra casuística nacional. 